\title{
Semi-classical Noise Treatment of Generated Supercontinuum Light by a Finite Energy Airy Pulse
}

\author{
A. Safaei ${ }^{\mathrm{a}}$ and Mohammad Agha Bolorizadeh \\ aDepartment of Photonics, Graduate University of Advanced Technology, Kerman, Iran \\ bDepartment of Physics, Yazd University, Yazd, Iran
}

Corresponding Author's Email: abolfazl_safaei@live.com

Received: Apr. 3, 2018, Revised: Jun. 9, 2018, Accepted: Jun. 19, 2018, Available Online: Dec. 27, 2019

DOI: 10.29252/ijop.13.2.127

\begin{abstract}
In this paper, a stochastic term is added to the classical generalized nonlinear Schrödinger Equation to describe the noise generated when light pulses propagate in a fiber. It has been shown that the generated supercontinuum light source fluctuates up to $50 \%$ of its output temporal intensity profile due to noises of different sources. The simulation method devised has been applied to the propagation of finite energy Airy pulse in a photonic crystal fiber and to study the generated noise.
\end{abstract}

KEYWORDS: Airy pulse, Light propagation, Noises, Supercontinuum generation.

\section{INTRODUCTION}

In order to study the nonlinear effects for the pulse propagation in an optical fiber, the nonlinear parameters of the medium as well as the intensity of the pulse should be high enough [1], [2]. The photonic crystal fibers (PCF), in comparison with conventional optical fibers, provide specific abilities such as working in a single mode from infrared to ultraviolet region [3]. Furthermore, implementing changes in the geometry of PCF structures, it is possible to change their nonlinear parameters as well as dispersion. As the light focuses into the core of photonic crystal fibers, their nonlinearity effects will be stimulated to further their applications.

There are numerous methods for finding the propagation parameters of optical fibers [2], such as beam propagation method [3], method of localized functions and finite elements method [4], in the literature. Due to the large variation in the effective refractive indices of holey optical fibers, specific methods can predict more accurate results for the propagation parameters as compared with other methods for different ranges of refractive index.

By establishing classical theory for nonlinear optics, it is known that the generation of new frequency components and spectral broadening are some of the inherent features of the nonlinear optics. There are a vast number of studies available in the field since 1970s [5]. The specific process in which an incident pulse with a narrow spectral width undergoes extreme broadening, generating an output with extensive width, is known as Supercontinuum Generation (SCG). SCG was reported in optical fiber by Alfano in 1999 [6] for the first time. SCG has many applications in microspectroscopy [7], microimaging [8], optical coherence tomography [9], [10] optical frequency metrology [11] and histopathology [12].

Supercontinuum process has a complicated mechanism whose thorough analysis is difficult. In accordance with this fact, many investigators have done comprehensive investigation on different mechanisms in SCG. Important mechanisms in supercontinuum process are the self-phase modulation (SPM) [13], the cross phase modulation (XPM) [14], the four wave mixing (FWM) [15], the 
stimulated Raman scattering (SRS) [16], the self-steepening (SS) [17] and the soliton fission [18]. Soliton fission is interpreted as the split-up of high-order solitons into multiple fundamental solitons leading to spectral broadening. Third order dispersion, selfsteepening and Raman scattering are the factors of soliton split up.

Generation of noise in fiber [19] or any other dielectric used to generate supercontinuum light source is the main cause of instability in it and, therefore, reducing its applicability in industry [20]. Depending on the parameters of the input pulse, the noises cause fluctuation in the supercontinuum generation spectrum [21]. There are many investigations available in the literature where the noises participate in this process are studied [21], [22]. The fundamental part of this noise has its root in quantum noise, which it is inherent in the nonlinear process leading to this kind of generated continuum light. This noise is modeled by adding a noise term in the generalized nonlinear Schrödinger equation (GNLSE), and we call it a semi-classical model, the term added to GNLSE, is a quantum noise, which has been phenomenologically sound term to be added to the classical GNLSE. In this paper, we simulate the supercontinuum generated in a PCF by a finite energy Airy beam [23]. The Airy beam was firstly observed in 1979 [24] and was exploited in optics [23] three decades later has vast interests and desirable features as Airy pulses resist dispersion and their dominant intensity peaks accelerate on propagation [25]-[29]. Also, Ament et al [29] studied, experimentally and theoretically, supercontinuum generation process making use of this type of pulse. When a symmetric and compact pulse is used to generate supercontinuum spectrum, generation of new frequency ends when the pulse is transformed into one or several fundamental soliton while by applying a finite energy Airy pulse the generation of new spectral components may continue after the dominant peak of the waveform has produced solitons [29].

\section{THEORY}

Taking into account a noise term, the supercontinuum generation process becomes a stochastic process [30], [31]. The stochastic generalized nonlinear Schrödinger equation, which is governing the supercontinuum process, is:

$$
\begin{aligned}
& \frac{\partial A(z, T)}{\partial z}+\frac{\alpha}{2} A(z, T)-\sum_{k=2}^{N} \frac{i^{k+1}}{k !} \beta_{k} \frac{\partial^{k} A(z, T)}{\partial T^{k}} \\
= & \xi(z, T) A(z, T)+i \gamma\left(1+i \frac{1}{\omega_{0}} \frac{\partial}{\partial T}\right) \times \\
& \left(A(z, T) \int R(t)|A(z, T-t)|^{2} d t\right),
\end{aligned}
$$

where $A(z, T), \beta_{k}, \alpha, \gamma$ and $\mathrm{R}(\mathrm{T})$ are the amplitude of the input pulse, the dispersion coefficient of $\mathrm{k}^{\text {th }}$ order, the loss coefficient, the nonlinear parameter and the response function of the medium, respectively. Here, also, $\xi(z, T)$ is a real Gaussian stochastic function [30] with the correlation relation as:

$$
\left\langle\xi\left(z_{1}, T_{1}\right) \xi\left(z_{2}, T_{2}\right)\right\rangle=\delta\left(z_{1}-z_{2}\right) \delta\left(T_{1}-T_{2}\right)
$$

The added noise term in Eq. 1 is the first approximation to quantum noise. There are other multiplicative noises which have quantum roots. One of them, $\xi(z, T)$, is related to the nonlinear parameter which has the correlation relations [32]:

$$
\begin{aligned}
& \left\langle\zeta\left(z_{1}, T_{1}\right) \zeta\left(z_{2}, T_{2}\right)\right\rangle= \\
& \quad \frac{\gamma\left(\omega_{0}\right)}{\omega_{0}} \delta\left(z_{1}-z_{2}\right) \delta\left(T_{1}-T_{2}\right),
\end{aligned}
$$

while the second one, $\eta(z, T)$, is related to Raman effect with the correlation relations [32]:

$$
\begin{aligned}
& \left\langle\eta\left(z_{1}, T_{1}\right) \eta\left(z_{2}, T_{2}\right)\right\rangle= \\
& \quad \gamma^{\prime \prime}\left(\omega_{0}\right)(n+1) \delta\left(z_{1}-z_{2}\right) \delta\left(T_{1}-T_{2}\right),
\end{aligned}
$$

where $\gamma^{\prime \prime}\left(\omega_{0}\right)$ and ' $n$ ' are second derivative of nonlinear parameter and number of photons, respectively. Note that $\gamma^{\prime}\left(\omega_{0}\right)=\gamma / \omega_{0}$. For the 
quantum Raman noise, it is evident from Eq. 4 that if there are no photons, there exists noise owing to spontaneous Raman effects. Corwin and his coworkers measured this noise [21]. In addition to these noises, there are other kinds of noises which correspond to the input pulse and the impurities of the media; i.e. changing refractive index randomly. We neglected the quantum Raman noise and simulated Eq. 1 employing the split step method.

To solve the stochastic NLSE (Eq. 1), we consider the fiber's parameters as in [5]. In order to take the Gaussian noise term into account, we applied the Box-Muller algorithm [33]. We study the propagation of a finite energy Airy beam through a photonic crystal fiber by considering:

$$
\mathrm{A}(\mathrm{z}=0, \mathrm{~T})=\sqrt{\mathrm{P}_{0}} \exp \left(\mathrm{aT} / \mathrm{T}_{0}\right) \operatorname{Ai}\left(\mathrm{T} / \mathrm{T}_{0}\right)
$$

where $\mathrm{P}_{0}$ and ' $\mathrm{a}$ ' are, respectively, the peak power of the beam and specific parameter of the Airy beam to ensure the Airy beam reaches finite energy, where the specific parameter of finite energy Airy beam should be $0<a<1$. Here, the properties of the Airy pulse are chosen from [29].

\section{NUMERICAL RESULTS}

Supercontinuum generation process is extensively studied by many groups [7], however there are few works on noises involved in this process [21], [34]. Here, in order to indicate that noise can affect deeply on the supercontinuum spectrum, we will simulate Eq. 1 with or without the noise term. As mentioned earlier, the simulation parameters for solving Eq. 1 are given in [5] and [29] for a photonic crystal fiber and a finite energy Airy pulse. Figure 1 shows the normalized field profile and the normalized intensity profile of a finite energy Airy pulse at the PCF input.

Firstly, we solve the Eq. 1 neglecting the stochastic term. From the practical considerations, the stability, the accuracy and the efficiency are the main issues to be handled in a numerical method for proper solution of the partial differential equation. For this reason, split step Fourier method is shown to be stable for solving GNLSE as well as the accuracy and efficiency. Figure 2 shows the evolution of the pulse inside the photonic crystal fiber in frequency and time domains. Figure 3 shows the wavelength profile at the output of the PCF, where the stochastic terms in Eq. 1 is eliminated.

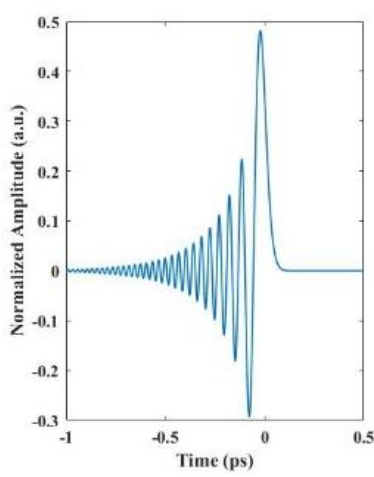

(a)

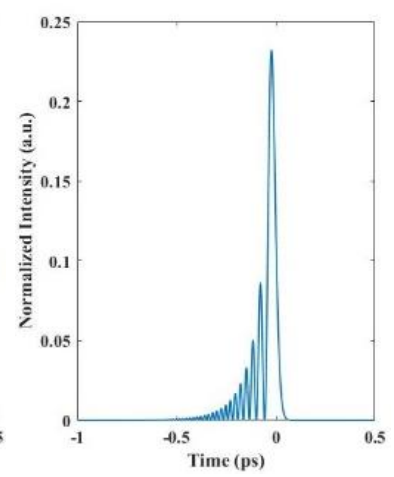

(b)
Fig. 1. The time dependence of a finite energy Airy pulse of Eq. 5 when a=0.11. Normalized field amplitude (a) and normalized intensity profile of this Airy beam (b) are shown.

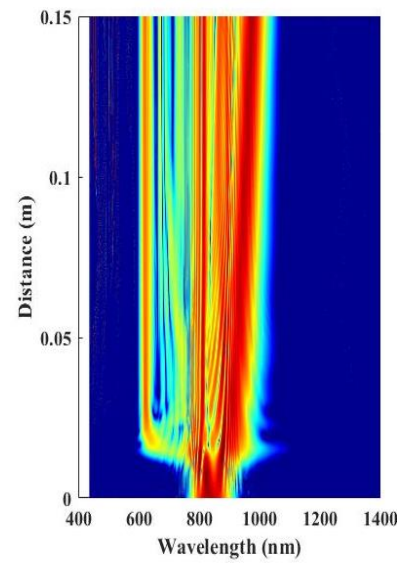

(a)

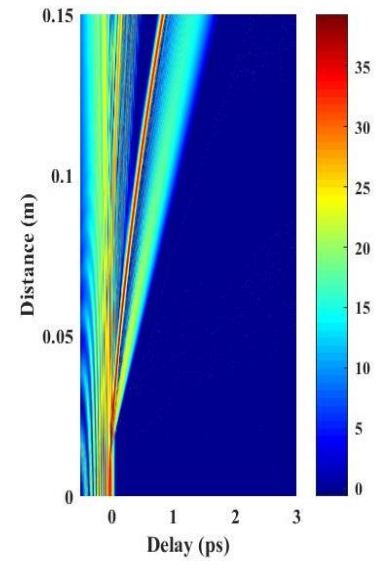

(b)

Fig. 2. Simulation results for the classical treatment of the SCG along the first $15 \mathrm{~cm}$ of a PCF fiber, when the stochastic term is not included. The spectra of light pulse versus wavelength are shown in (a), while the delay $(+/-)$ with respect to the group velocity of the generated SCG is shown in (b). 


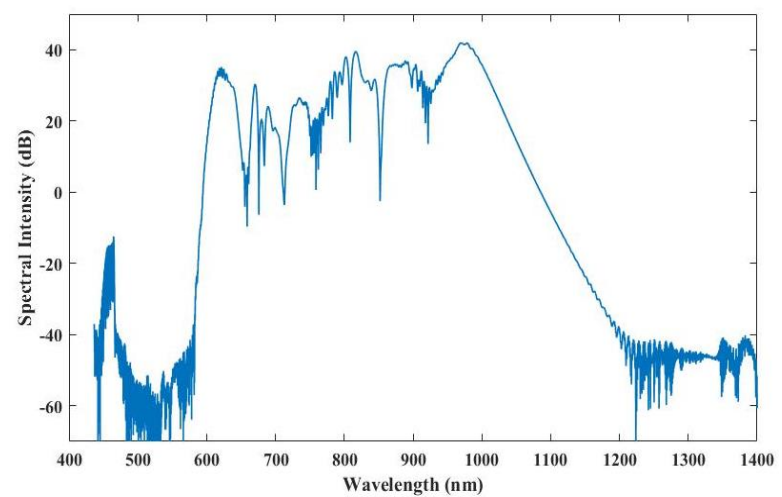

Fig. 3. The expected spectrum of light pulse versus wavelength at the output of a $15 \mathrm{~cm}$ PCF employing a classical treatment of noise in SCG is simulated where the noise term was eliminated.

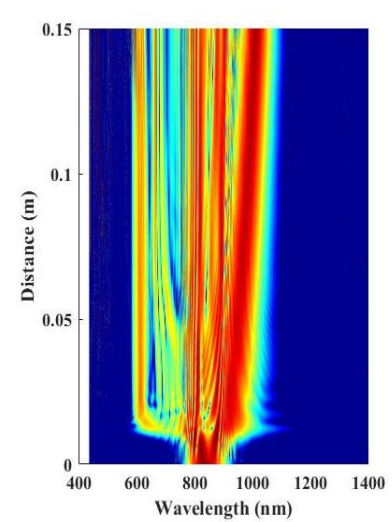

(a)

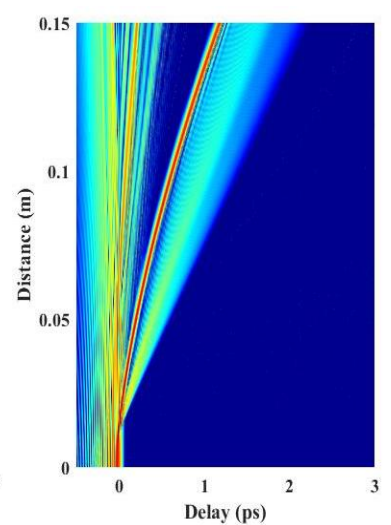

(b)
Fig. 4. Simulation results for the semi-classical treatment of the SCG along the first $15 \mathrm{~cm}$ of a PCF fiber, when the stochastic term is included. The spectra of light pulse versus wavelength are shown in (a), while the delay (+/-) with respect to the group velocity of the generated SCG is shown in (b).

Now, an attempt was made to study the effect of noise on the supercontinuum spectrum. Therefore, we solve the Eq. 1 when the stochastic terms are included. We have made use of the Box-Muller algorithm to generate a real Gaussian stochastic function and integrate Eq. 1 by split step method. Figures 4 and 5 show the simulation results for Eq. 1 in the presence of stochastic terms. The evolution of the light pulse along the PCF and the wavelength profiles at the output are presented in Figs. 4 and 5, respectively.

The two simulations (with and without stochastic noise terms) show that how stochastic noises affect the supercontinuum process. The stochastic terms create fluctuations in the output profiles. Also, the differences between two simulations can be varied depending on the parameters of the input pulse. So, the fluctuations make the generated supercontinuum light by Airy pulse to be noisy for some of its applications. Soliton self-frequency shift phenomena occur in supercontinuum generation process and have applications in technology particularly when one applies to Airy pulse (the solitons which created in PCF may open a new application). These fluctuations in the vicinity of the created solitons will be at odds for new applications.

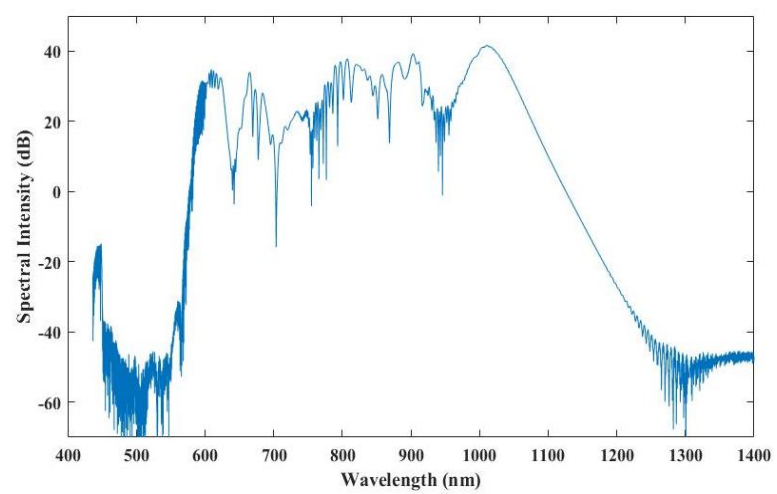

Fig. 5. The same as Fig. 3, but for the semi-classical treatment of noise in SCG when the stochastic term was included.

\section{CONCLUDING REMARKS}

Currently, the applicability of supercontinuum light sources is known to scientific and technological communities. However, these new light sources suffer from noises which could limit their applications. Part of these noises is inherent quantum noises. In this paper, in order to show these noises in final supercontinuum spectrum from an Airy pulse, we added, phenomenologically, a stochastic term to the GNLSE. Then, we simulated the GNLSE with and without this stochastic term. To simulate Eq. 1 with stochastic term, the Box-Muller algorithm is applied to generate stochastic function and integrate Eq. 1 by split step method. As we expected, our simulation results indicated differences at the output. As an example, the semi-classical model 
(Fig. 4(b)) predicts longer delay for the formed soliton as compared with the classical result of Fig. 2(b). Also, the soliton fission occurs at the shorter travel distance of the pulse along the fiber for the semi-classical model with respect to the classical model. Although, the noises exist in supercontinuum generation spectrum, a detailed knowledge about their source and their treatment is needed to be reduced and/or to be engineered in favor of technologies. We speculate that a quantum mechanical treatment is needed for pulses as short as $1 \mathrm{ps}$ in order to study these noises in supercontinuum spectrum. There are several quantum theories [34]-[36] for pulse propagation along optical fiber in literature, but none of them are suitable for supercontinuum generation process. We believe that by using a powerful quantum scheme, the scientists and engineers can be fully exploited supercontinuum light in all its capacities. Because, the quantum theory enables us to control the fluctuations and the fluctuations in vicinity of the solitons can be squeezed.

\section{REFERENCES}

[1] M. Farries, P.ST.J. Russell, M.E. Fermann, and D.N. Payne, "Second harmonic generation in an optical fiber by self-written $\chi^{2}$ grating," Electron. Lett. Vol. 23, pp. 322-324, 1987.

[2] H.M. Masoudi and J.M. Arnold, "Modeling second-order nonlinear effects in optical waveguides using a parallel-processing beam propagation method," IEEE J. Quant. Electron. Vol. 31, pp. 2107-2113, 1995.

[3] K. Bush and S. Lolkes, Photonic Crystal Advance in Design, Fabrication and Characterization, New York: Wiley, 2004.

[4] M. Koshiba, K. Hayata, and M. Suzuki, "Improved finite-element formulation in terms of the magnetic-field vector for dielectric waveguide," IEEE Trans. Microw. Theory Tech. Vol. 33, pp. 227-233, 1985.

[5] A. Safaei Bezgabadi, M.A. Bolorizadeh, and A. Zakerifar, "Effect of higher order dispersion on supercontinuum spectrum," Proc. SPIE, Vol. 9586, pp. 95860Y (1-6), 2015 and references therein.
[6] J. Herrmann, U. Griebner, N. Zhavoronkov, A. Husakou, D. Nickel, J.C. Knight, W.J. Wadsworth, P.St.J. Russell, and G. Korn, "Experimental evidence for supercontinuum generation by fission of higher-order solitons in photonic fibers," Phys. Rev. Lett. Vol. 88, pp. 173901 (1-4), 2002.

[7] J.M. Dudley and J.R. Taylor, Supercontinuum Generation in Optical Fibers, Cambridge: Cambridge University Press, $3^{\text {rd }}$ Ed, 2010.

[8] X. Li, D. Zhu, Z. Ma, L. Pan, D. Wang, and J.H Wang, "Feasibility study of the detection of chlorpyrifos residuals on apple skin based on infrared micro-imaging," Opt. Eng. Vol. 51, pp. 103204(1-11), 2012.

[9] J.G. Fujimoto, "Optical coherence tomography for ultrahigh resolution in vivo imaging," Nature Biotech. Vol. 21, pp. 1361-1367, 2003.

[10]W. Drexler, "Ultrahigh-resolution optical coherence tomography," J. Biomed. Opt. Vol. 9, pp. 47-74, 2004.

[11]T. Udem, J. Reichert, R. Holzwarth, and T.W. Hänsch, "Accurate measurement of large optical frequency differences with a modelocked laser," Opt. Lett. Vol. 24, pp. 881-883, 1999.

[12]T. Tu, Y. Liu, D. Turchinovich, M. Marjanovic, J.K. Lyngsø, J. Lægsgaard, E.J. Chaney, Y. Zhao, S. You, W.L. Wilson, B. $\mathrm{Xu}, \mathrm{M}$. Dantus, and S.A. Boppart "Stain-free histopathology by programmable supercontinuum pulses," Nat. Photonics, Vol. 10, pp. 534-540, 2016.

[13]R.H. Stolen and C. Lin, "Self-phasemodulation in silica optical fibers," Phys. Rev. A, Vol. 17, pp. 1448-1458, 1978.

[14] G.P. Agrawal, Fiber-Optic Communication Systems, New York: Wiley, $2^{\text {nd }} E d, 2007$.

[15] J.M. Dudley, G. Genty, and S. Coen, "Supercontinuum generation in photonic crystal fiber," Rev. Mod. Phys. Vol. 78, pp. 1135-1184, 2006.

[16]B.E.A. Saleh and M.C. Teich, Fundamentals of Photonics, New Jersey: Wiley, 2007.

[17]R.R. Alfano, The Supercontinuum Laser Source, New York: Springer, 2006.

[18]A. Hasegawa and S. Matsumoto, Optical Solitons in Fibers, Heidelberg: Springer, 2003. 
[19]P.D. Drummond and J.F. Corney, "Quantum noise in optical fibers. I. Stochastic equations," J. Opt. Soc. Am B, Vol. 18, pp. 139-152, 2001.

[20] L. Hollberg, C.W. Oates, E.A. Curtis, E.N. Ivanov, S.A. Diddams, T. Udem, H.G. Robinson, J.C. Bergquist, R.J. Rafac, W.M. Itano, R.E. Drullinger, and D.J. Wineland, "Optical frequency standards and measurements," IEEE J. Quantum Electron. Vol. 37, pp. 1502-1513, 2001.

[21] K.L. Corwin, N.R. Newbury, J.M. Dudley, S. Coen, S.A. Diddams, K. Weber, and R.S. Windeler, "Fundamental noise limitations to supercontinuum generation in microstructure fiber," Phys. Rev. Lett. Vol. 90, pp. 113904 (1-16), 2003.

[22] Y. Liu, Y. Zhao, J. Lyngsø, S. You, W.L. Wilson, H. Tu, and S.A. Boppart "Suppressing short-term polarization noise and related spectral decoherence in all-normal dispersion fiber supercontinuum generation." J. Lightwave Technol. Vol. 33, pp. 18141820, 2015.

[23] G.A. Siviloglo, and D.N. Christodoulides, "Accelerating finite energy Airy beams," Opt. Lett. Vol. 32, pp. 979-981, 2007.

[24] M.V. Berry and N.L. Balazs, "Non-spreading wave packets," Am. J. Phys. Vol. 47, pp. 264267, 1979.

[25] J. Baumgart, M. Mazilu, and K. Dholakia, "Optically mediated particle clearing using Airy wave packets," Nat. Photon. Vol. 2, pp. 675-678, 2008.

[26]P. Polynkin, M. Kolesik, J.V. Moloney, G.A. Siviloglou, and D.N. Christodoulides, "Curved plasma channel generation using ultra-intense Airy beams," Science, Vol. 324, pp. 229-232, 2009.

[27]R. Bekenstein, R. Schley, M. Mutzafi, C. Rotschild, and M. Segev, "Optical simulations of gravitational effects in the NewtonSchrödinger system," Nat. Physics, Vol. 11, pp. 872-878, 2015.

[28]M. Shen, J. Gao, and L. Ge, "Solitons shedding from Airy beams and bound states of breathing Airy solitons in nonlocal nonlinear media," Sci. Rep. Vol. 5, pp. 9814 (1-5), 2015.

[29]C. Ament, P. Polynkin, and J.V. Moloney, "Supercontinuum generation with femtosecond self-healing Airy pulses," Phys. Rev. Lett. Vol. 107, pp. 243901 (1-5), 2011.

[30]E. Koleden and E. Platen, Numerical solution of stochastic differential equations, Heidelberg: Springer-Verlag Berlin, 1992.

[31]T. Sauer, "Computational solution of stochastic differential equations," WIREs Comput. Stat. Vol. 5, pp. 362-371, 2013.

[32]P.D. Drummond and M. Hillery, The Quantum Theory of Nonlinear Optics, New York: Cambridge Press, 2014.

[33] W.J. Thistleton, J.A. Marsh, K. Nelson, and C. Tsallis, "Generalized Box-Muller method for generating -Gaussian random deviates," IEEE Trans. Inf. Theory, Vol. 53, pp. 4805-4810, 2007.

[34]A. Safaei Bezgabadi and M.A. Bolorizadeh, "Quantum mechanical treatment of the third order nonlinear term in NLS equation and the supercontinuum generation," Proc. SPIE, Vol. 9958, pp. 995803 (1-7), 2016.

[35] A. Safaei, A. Bassi, and M.A. Bolorizadeh, "Quantum treatment of field propagation in a fiber near the zero dispersion wavelength" J. Opt. Vol. 20, p. 055402, 2018.

[36] A. Safaei Bezgabadi and M.A. Bolorizadeh, "Quantization of electromagnetic radiation in dielectrics with presence of third order dispersion term," arXiv: 1801.02098 [Phys. Opt. pp. 1-14, 2018.

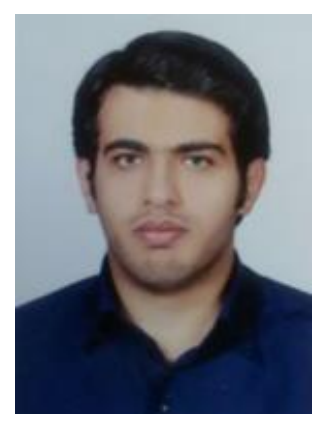

Abolfazl Safaei was born in Yazd, Iran, on September 1, 1987. He received B.Sc. degree in Physics at the Yazd University and M.Sc. degree at the Graduate University of Advanced Technology (GUAT), minors in atomic and molecular physics on June 2009 and photonics on December 2012, respectively. Currently, he is studying $\mathrm{PhD}$ degree in atomic and molecular physics at the GUAT, Kerman, Iran. 
He received a scholarship from The Ministry of Science, Research and Technology for his $\mathrm{PhD}$ program. Mr Safaei is a member of Iranian Physical Society. His research interests include the quantum theory of nonlinear optics, quantum field theory, supercontinuum generation in optical fibers, atom optics and Bose-Einstein condensation.

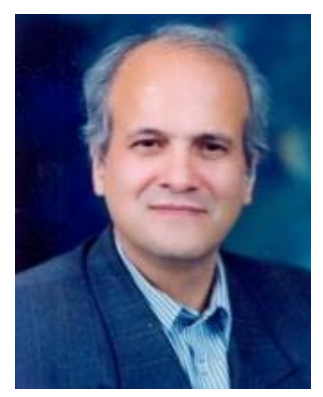

Mohammad Agha Bolorizadeh started his career at the Shahid Bahonar University of
Kerman, Kerman, Iran as assistant professor in 1984. He later (in 2009) joined the Graduate University of Advanced Technology (GUAT), Mahan, Kerman, Iran.

He had joined with the Atomic and Molecular Group at the Queen's University of Belfast in 1992, twice with the Flinders University of South Australia, Adelaide, SA, Australia on 1997 and 2005 as well as the Physics Department at the University of Western Ontario, London, Ontario, Canada in 2000 as a visiting professor. His topics of research are scattering problems in atomic, molecular and optical areas as well as optical communication and applications of fibers in industry and medicine. Prof. Bolorizadeh is a member of the Optical Society of America, a member of Iranian Physical Society and a member of the Optics and Photonics Society of Iran (OPSI). 
THIS PAGE IS INTENTIONALLY LEFT BLANK. 